\title{
A HIGH RESOLUTION FAR-UV SPECTRAL ATLAS OF CSPNs AND HOT WHITE DWARFS
}

\author{
R.W. TWEEDY \\ Steward Observatory, University of Arizona, Tucson, USA
}

A high-resolution IUE spectral atlas of central stars of planetary nebulae and hot white dwarfs has been produced (part of Tweedy, 1991, PhD thesis from the University of Leicester, UK), and examples from it are shown here. It has been sorted into an approximate evolutionary sequence, based on published spectroscopic analyses, from the cool $28,000 \mathrm{~K}$ young central star He 2-138, through the hot objects like NGC 7293 and NGC 246 at $90,000 \mathrm{~K}$ and $130,000 \mathrm{~K}$ respectively, down to $40,000 \mathrm{~K}$ DA white dwarfs like GD 2, which is the chosen cutoff for this selection. Copies of a revised version of this atlas, which will include more recent spectroscopic information and also white dwarfs down to $35,000 \mathrm{~K}$ - to include the Si III object GD 394 - will be sent to anyone who requests one. 